\title{
Gene expression profiling of familial and sporadic pituitary adenomas S Barry ${ }^{1}$, J A Saleh ${ }^{1}$, E Gadaleta ${ }^{2}$, C Chelala ${ }^{2}$ and M Korbonits ${ }^{1}$ \\ ${ }^{1}$ Department of Endocrinology, William Harvey Research Institute \\ ${ }^{2}$ Centre for Molecular Oncology, Barts Cancer Institute,
}

Barts and The London School of Medicine and Dentistry, Queen Mary University of London, London EC1M 6BQ, UK

\section{Introduction}

Familial isolated pituitary adenoma (FIPA) is an autosomal dominant condition with incomplete penetrance. Heterozygote mutations have been identified in the arylhydrocarbon receptor interacting protein (AIP) gene in $20 \%$ of FIPA families. In AIP positive patients, the disease is occurring at a younger age and have larger, more aggressive tumours than AIP negative patients and often show invasion at the time of diagnosis as well as poor response to somatostatin analogues than sporadic tumours ${ }^{1,2}$

\section{Aims}

The aim of this study was to perform comparative gene expression microarray analysis of familial AIP positive and AIP negative adenomas and compare them to sporadic tumours and normal pituitary to discover novel genes and pathways responsible for familial pituitary tumorigenesis. 3. Methods

We have performed gene expression analysis on normal pituitary, sporadic GH-secreting adenomas, AIP positive and AIP negative familial somatotroph adenomas (five samples of each category) using the Affymetrix human Gene Chip HG-U133 Plus 2.0 array. Data analysis was carried out in the statistical ' $R$ ' environment. Ingenuity Pathway Analysis (IPA) tool was used for pathway analysis. Expression of the ten selected genes from microarray analysis was validated by quantitative reverse transcriptase PCR. Functional assays were performed using BioCoat-Matrigel invasion chambers.

\section{Unsupervised hierarchical clustering}

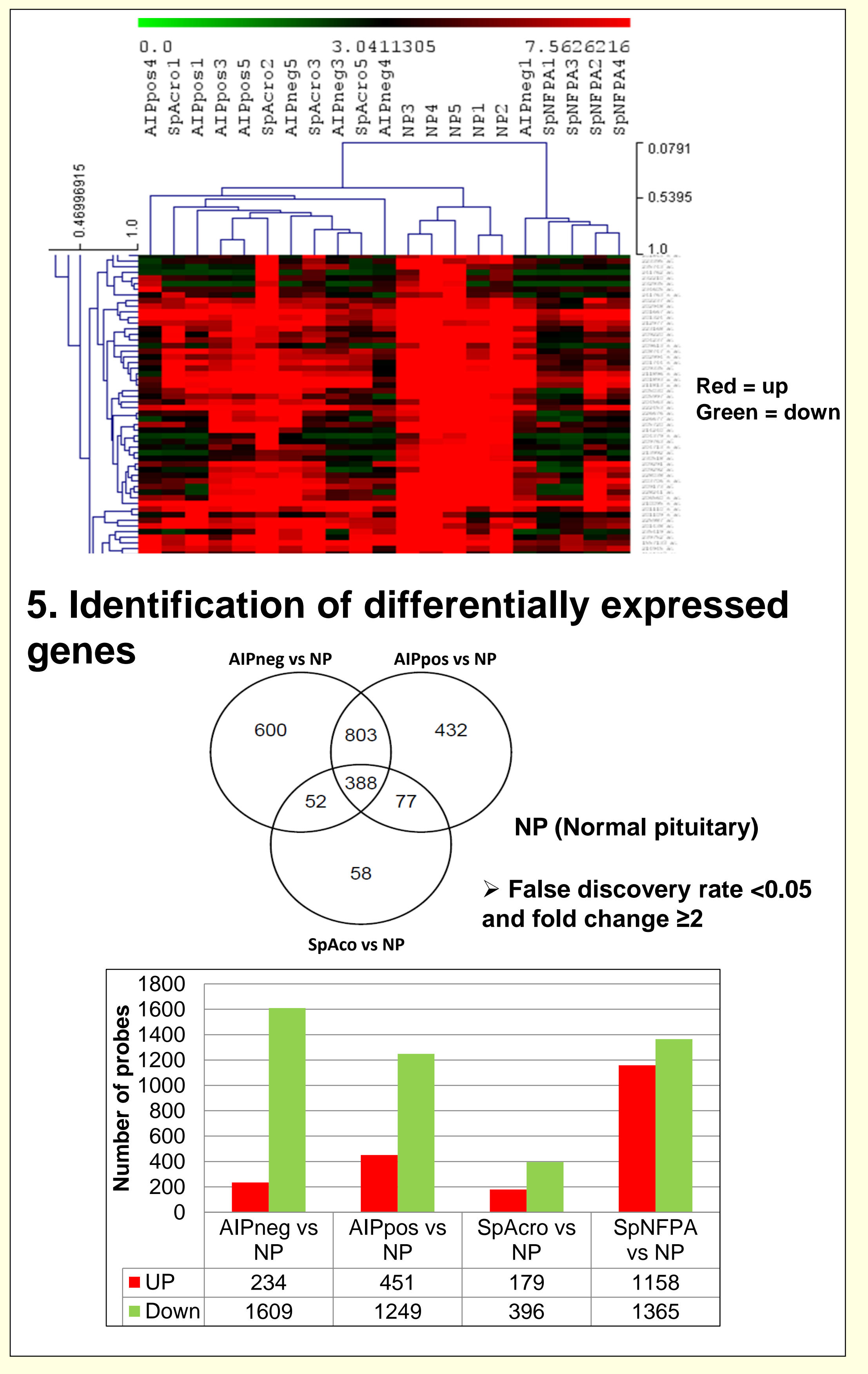

\section{Ingenuity Pathway Analysis}

Ingenuity pathway analysis shows the top functional modules and top canonical pathways

$\overline{\text { im AIPposvsAIPneg- }} \overline{\equiv \text { AIPposvsAcro- }} \overline{\text { AIPnegvsAcro- }}$
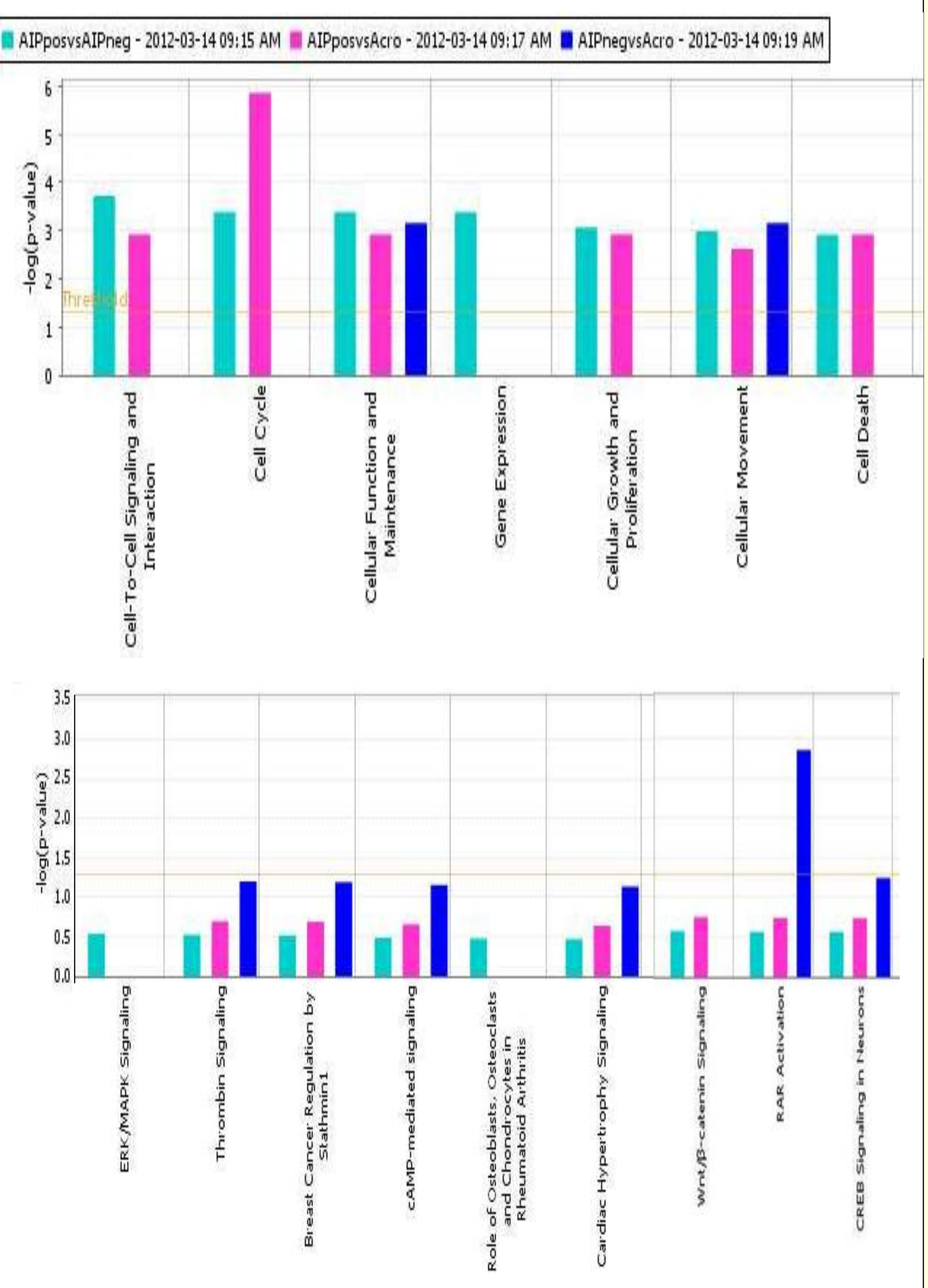

\section{Validation by RT-qPCR}

Five up (S100P, NTS, SPP1, CRTAC1, ZEB1) and five down-regulated (CTNNB1, CDH1, ESRP1, PERP, EPCAM) genes were validated
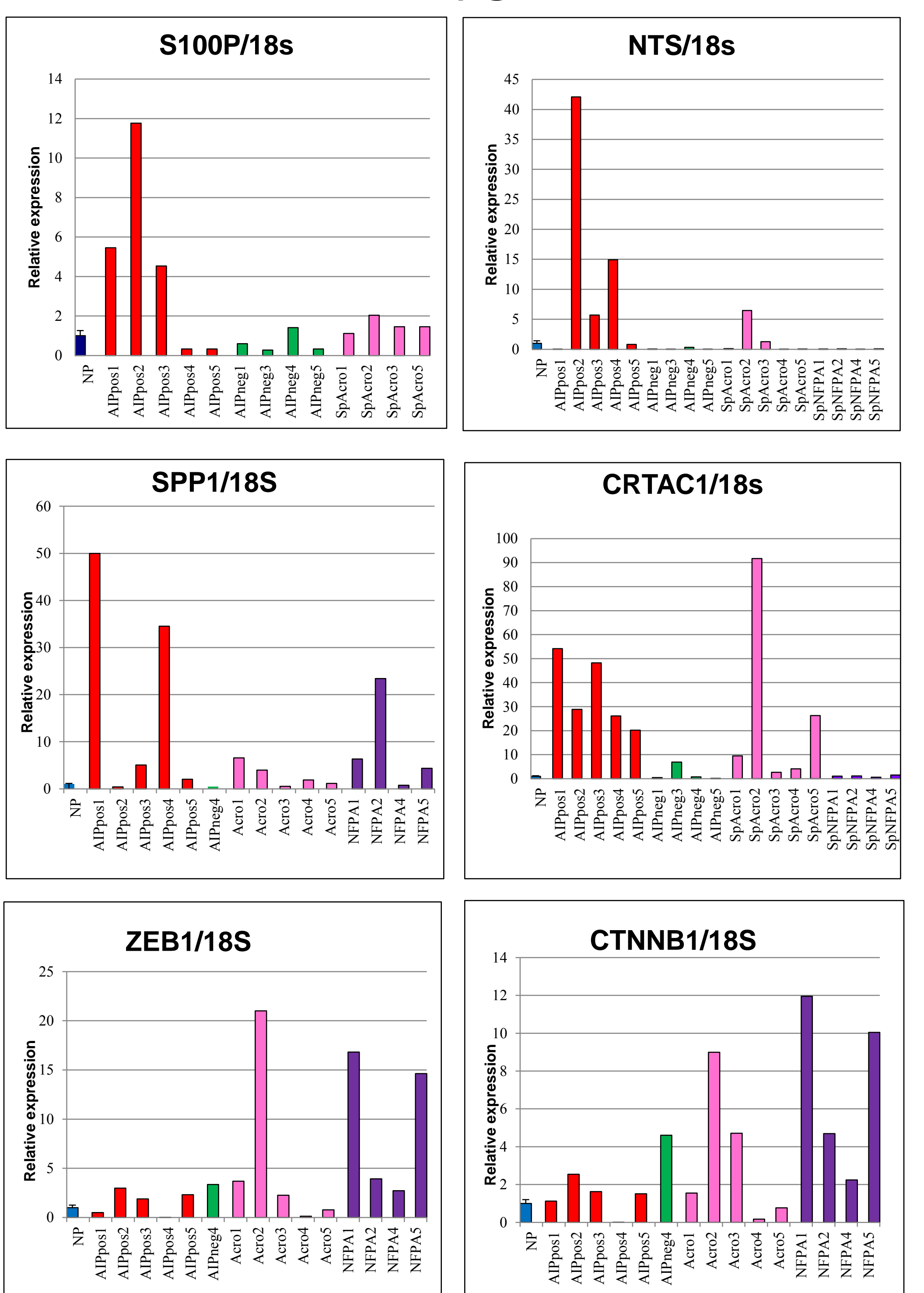

CTNNB1/18S

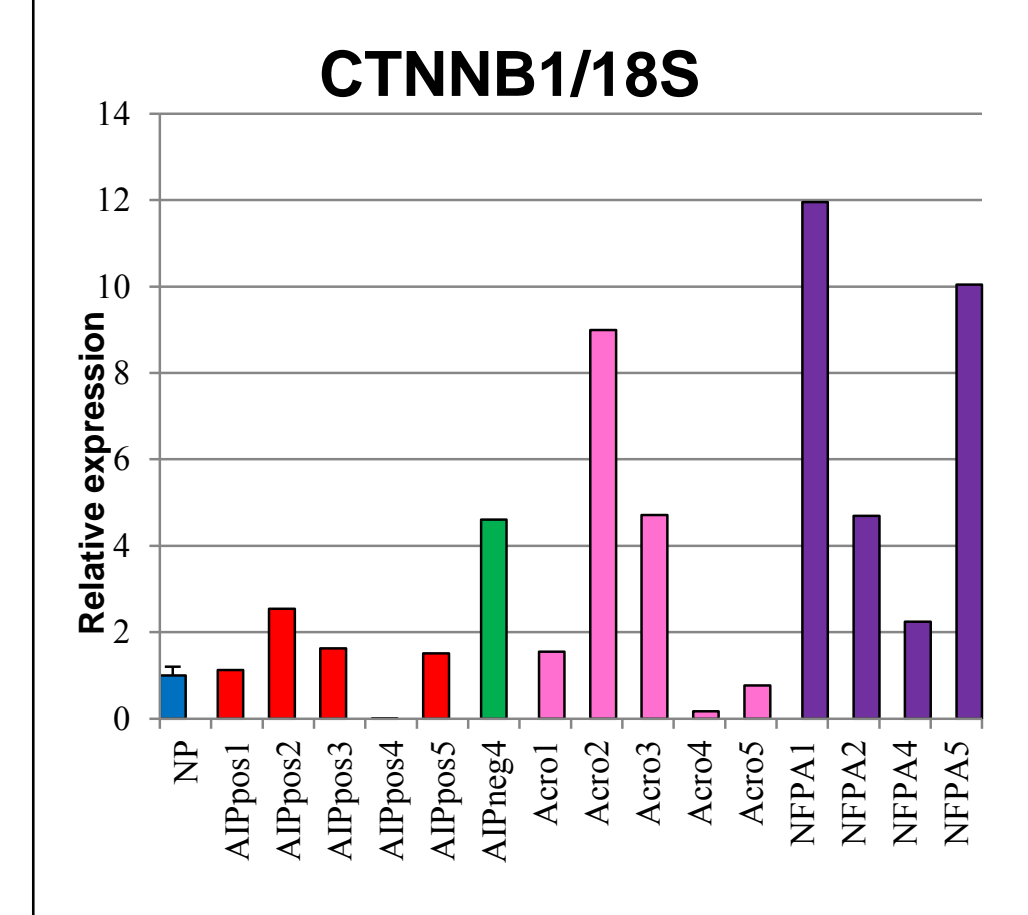

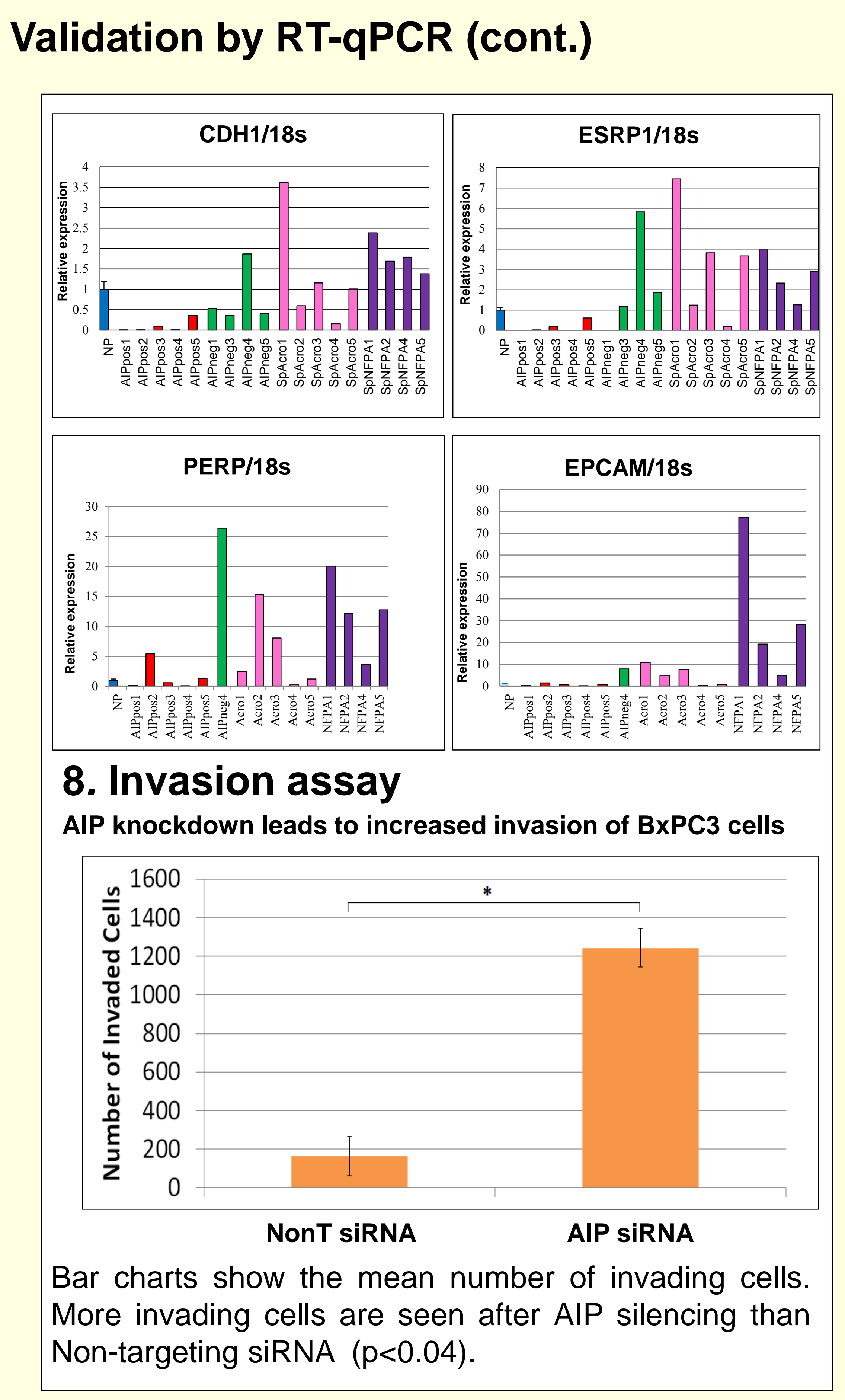

\section{Conclusions}

We have identified a large number of differentially expressed genes in pituitary adenomas compared to normal pituitary. In addition, a small number of genes differ in their expression levels between familial AIP positive and sporadic adenomas. These genes are involved in epithelial-to-mesenchymal transition $(\mathrm{CDH} 1$, ESRP1, EPCAM, PERP, CTNNB1, ZEB1) and in invasion pathway (S100P, SPP1, NTS).

RT-qPCR data of the increased expression of mesenchymal marker, invasive markers and the decreased expression of epithelial markers were consistent with the microarray data.

These results indicate that these transcriptional changes likely reflect the clinically seen more aggressive phenotype in AIP positive patients. In pituitary tumorigenesis EMT likely occurs within a specific genetic context and may be related to their increased local invasion and more aggressive behaviour. We have also demonstrated that lack of AIP plays a critical role in cellular invasion. Therefore, different pathways in pituitary adenoma progression exist.

\section{References}

1 Daly AF, et al (2010) J.Clin.Endocrinol.Metab., 95, E373-E383. 2. Chahal, H, et al (2012) J.Clin.Endocrinol.Metab., 97, E1411-1420.

11. Acknowledgement

We are grateful for the financial support from Pfizer to our studies related to FIPA. 